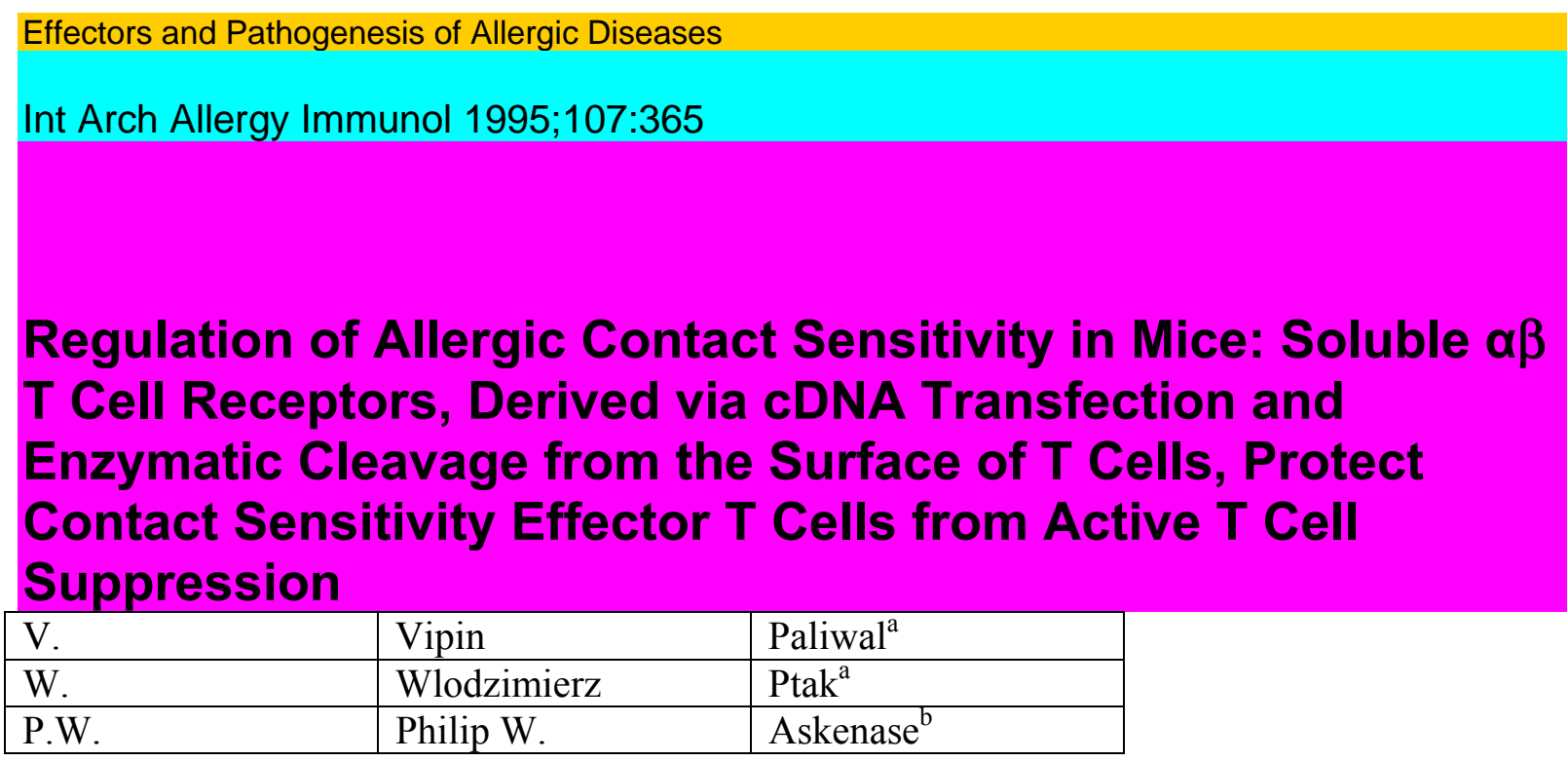

a Jagiellonian University School of Medicine, Krakow, Poland; and ${ }^{\text {b} Y a l e ~ U n i v e r s i t y ~}$ School of Medicine, New Haven, Conn., USA

\title{
Key Words
}

Suppressor T cells

Contrasuppressor factor

Soluble TCR

TCR cDNA Transfection

Correspondence to: Dr. Philip W. Askenase, Section of Allergy and Clinical Immunology, 904 LCl, Yale University, Box 208013, New Haven, CT 06520-8013 (USA)

Using a system in which suppressor T cells actively suppressed contact sensitivity effector T cell adoptive cell transfers, in vitro pre-treatment of the effector cells with soluble $\mathrm{T}$ cell receptor (sTCRs) protected the contact sensitivity effector $\mathrm{T}$ cells from the active $\mathrm{T}$ cell suppression. sTCRs were derived from BWE5147 thymoma cells that were previously transfected with chimeric cDNAs encoding the extracellular domains of the $\alpha$ and $\beta$ chains of conalbuminspecific TCR from the Th2 DIO CD4+ T cell clone that were spliced to the glycosylphosphatidylinositol (GPI) membrane anchor sequences of the Pl-linked molecule, Thy-1. Treatment of TCR $\alpha ß$-transfected cells with Pi-specific phospholi-pase C (PI-PLC) caused release of the transfected TCR in soluble form from the cell surface. sTCR protection from suppression of contact sensitivity effector T cells was not due to PI-PLC contaminating the sTCRs, since treatment of DIO TCR $\alpha ß$-expressing BW5147 cells with PI-PLC alone had no effect, and sTCR purified further on anti-TCRß (H57) monoclonal antibody columns preserved contact-sensitivityassisting activity. Only TÇR-Thy-1-cDNA-transfected cells, but not sev-

eral other cell lines, including cells transfected with the DIO TCR $\alpha \beta$ cDNA, but with the normal transmembrane portion, produced contact-sensitivity-protecting activity.

Specific antigen (conalbumin) of the DIO TCR did not interfere with the protecting activity of DIO sTCR. The contact-sensitivity-protecting activity of sTCR was shown to adhere to and be subsequently eluted from anti-TCR $\alpha$ (H28) and anti-TCRß (H57) monoclonal antibody columns. Reduced and separated TCR $\alpha \beta$ chains of DIO sTCR lacked contact-sensitivity-protective activity, but subsequent recombination by covalent joining of both chains and heterodimer formation, via the oxido-shuffling reaction, restored activity. 
We concluded that DIO sTCR, derived via cDNA transfection of chimeric TCR Thy-1 constructs, and PI-PLC release from cell surfaces, could protect contact sensitivity effector $\alpha \beta \mathrm{T}$ cells from $T$ cell suppression, and thus was contrasuppressive. The contrasuppressive activity was not antigen specific and required both chains of TCR $\alpha \beta$, and their disulfide linkage, to express this activity.

(C) 1995 S.KargerAG, Basel 1018-2438/95/1073-0365 \$8.00/0 